Cinémas

Revue d'études cinématographiques

Journal of Film Studies

\title{
De la critique de cinéma à la critique de film : la modernité antonionienne, effet de critique ou démarche d'auteur?
}

\section{Dominique Nasta}

Volume 6, numéro 2-3, printemps 1996

La Critique cinématographique

URI : https://id.erudit.org/iderudit/1000971ar

DOI : https://doi.org/10.7202/1000971ar

Aller au sommaire du numéro

Éditeur(s)

Cinémas

ISSN

1181-6945 (imprimé)

1705-6500 (numérique)

Découvrir la revue

Citer cet article

Nasta, D. (1996). De la critique de cinéma à la critique de film : la modernité antonionienne, effet de critique ou démarche d'auteur? Cinémas, 6(2-3), 45-64. https://doi.org/10.7202/1000971ar
Résumé de l'article

Cet article essaie de mettre en évidence la dualité constitutive auteur/critique, principal embrayeur de la modernité antonionienne. La critique des films d'Antonioni s'avère difficile, car il s'agit de juger une oeuvre qui véhicule un message imprégné de multiples thèmes socioculturels, tout en rompant avec la tradition classique du récit filmique. Un panorama de critiques sur L'Avventura suivi d'un survol des opinions du cinéaste permettent ici de mieux cerner la problématique visée. 


\title{
De la critique de cinéma à la critique de film: la modernité antonionienne, effet de critique ou démarche d'auteur ? ${ }^{1}$
}

\section{Dominique Nasta}

\begin{abstract}
RÉSUMÉ
Cet 'article essaie de mettre en évidence la dualité constitutive auteur/critique, principal embrayeur de la modernité antonionienne. La critique des films d'Antonioni s'avère difficile, car il s'agit de juger une œuvre qui véhicule un message imprégné de multiples thèmes socioculturels, tout en rompant avec la tradition classique du récit filmique. Un panorama de critiques sur L'Avventura suivi d'un survol des opinions du cinéaste permettent ici de mieux cerner la problématique visée.
\end{abstract}

\section{ABSTRACT}

This article explores the constitutive duality of author and critic, the primary impetus behind Antonioni's modernity. Criticizing his films is difficult because it involves judging work that not only carries a message implicated in multiple socio-cultural themes, but which at the same time breaks with the classic tradition of film plot. An overview of criticism of L'Avventura, followed by the filmmaker's own opinions, will permit a better understanding of the problematic under study.

En paraphrasant le titre d'une remarquable étude publiée il y a quelques années sur Michelangelo Antonioni, Identificazione di un autore (lui-même paraphrase d'Identificazione di una donna), il s'agira, dans les pages qui suivent, d'essayer 
d'identifier à travers un vaste panorama de la critique les sources d'un phénomène qui demeure unique, la modernité antonionienne ${ }^{2}$.

Le cinéma d'Antonioni et ce qu'il subsume, à savoir ses films, ont provoqué une véritable révolution dans le monde de la critique cinématographique.

Une première explication de ce phénomène se trouve, bien évidemment, au cœur même de l'œuvre antonionienne; il s'agit de la traduction en images d'un monde arbitraire, qui assouplit la raideur absolue du discours classique. Le constat d'une situation non finalisable, la relativité des rapports humains, le refus de recourir aux effets, la création d'une nouvelle architecture visuelle, ce sont là des notions "installées" dès le premier long métrage d'Antonioni. Mais ce n'est qu'avec la sortie, 10 ans plus tard, de L'Avventura (1960) que le phénomène critique prend de l'envergure: c'est la raison pour laquelle nous avons choisi de nous pencher sur ce film plutôt que de tenter un parcours globalisant, qui risquerait de manquer d'exhaustivité.

Une deuxième justification du phénomène relève plutôt du contexte socio-esthétique dans lequel se situe Antonioni en tant que cinéaste, penseur visuel, lui-même ancien critique littéraire et cinématographique. L'après-guerre offre au monde une image de désenchantement, de perte des illusions; ceci se traduira par la nécessité de l'élaboration d'une nouvelle réalité filmique, celle prônée par les néo-réalistes italiens et ultérieurement, d'une manière quelque peu différente, par la Nouvelle Vague française. Parallèlement apparaît aussi une nouvelle génération de critiques où se bousculent écrivains, philosophes, sociologues, mais surtout des jeunes cinéastes qui écrivent sur le cinéma des autres, alors qu'ils sont en train d'élaborer leur propre grammaire filmique.

La critique journalière, hebdomadaire ou mensuelle de la fin des années 50 et du début des années 60 abandonne, du moins pour quelque temps, le sillage impressionniste pour devenir "expressionniste", se positionnant à un niveau d'analyse qui la rattache plus que jamais aux théories littéraires en vogue. Ce passage à une nouvelle forme de critique n'est évidemment pas généralisé, car comme on le verra plus bas, les quotidiens français ou 
allemands à vocation populaire n'abandonnent pas leurs vieux réflexes, au risque de voir diminuer leur nombre de lecteurs. Certaines revues d'outre-Atlantique iront même juqu'à rejeter en bloc "les modernes» jugés pour la plupart comme irresponsables, imposteurs, dépourvus de toute morale.

Le public, lui aussi, a changé. Le cinéma, désormais incorporé à certains cursus d'études en France, en Italie et davantage encore aux États-Unis, ajoute à son étiquette de "divertissement" celle de l'enrichissement psycho-esthétique. Un vieux rêve nourri par les avant-gardes du début du siècle, celui de la pensée visuelle active, semble enfin s'accomplir. Cet accomplissement se double d'une reconnaissance des influences de la nouvelle littérature (qu'il s'agisse de Joyce, Woolf, Svevo ou de leurs héritiers français Duras, Robbe-Grillet, etc.) par un public averti, soucieux d'établir des équivalences entre le lu et le vu. Les critiques et les cinéastes, Antonioni en tête, vont largement contribuer à cette validation du cinéma en tant qu'objet esthétique, pas toujours facile d'abord, souvent déconcertant, voire même dérangeant, mais somme toute passionnant.

Film manifeste malgré lui, L'Avventura, sorti bien après les premières grandes réalisations de Bergman, un an après Hiroshima mon amour (Resnais, 1959), et en même temps que La Dolce Vita (qui "sauve" l'Italie à Cannes en cette année 1960), fait brusquement oublier ses illustres prédécesseurs et ses contemporains, du moins pendant quelque temps. Grâce aux critiques, Antonioni finit par gagner une rude bataille à Cannes, le public lui étant largement défavorable.

Une vaste entreprise de validation de son film et par conséquent du type de cinéma qu'il véhicule s'enclenche partout en Europe; par la force des choses, ce sont les exégètes italiens qui, les premiers, démontrent que tout était déjà dit dans le premier long métrage d'Antonioni. C'est vrai, les signes extérieurs de Cronaca di un amore étaient plus trompeurs que ceux de $L ' A v$ ventura: il s'agissait d'un mélodrame ayant tous les ingrédients du genre, alors que le récit de L'Avventura se présente comme un policier à l'envers, un récit déplacé, complètement dédramatisé. Les revues italiennes soulignent également l'importance des propos que le cinéaste lui-même a véhiculés sur son travail et sur 


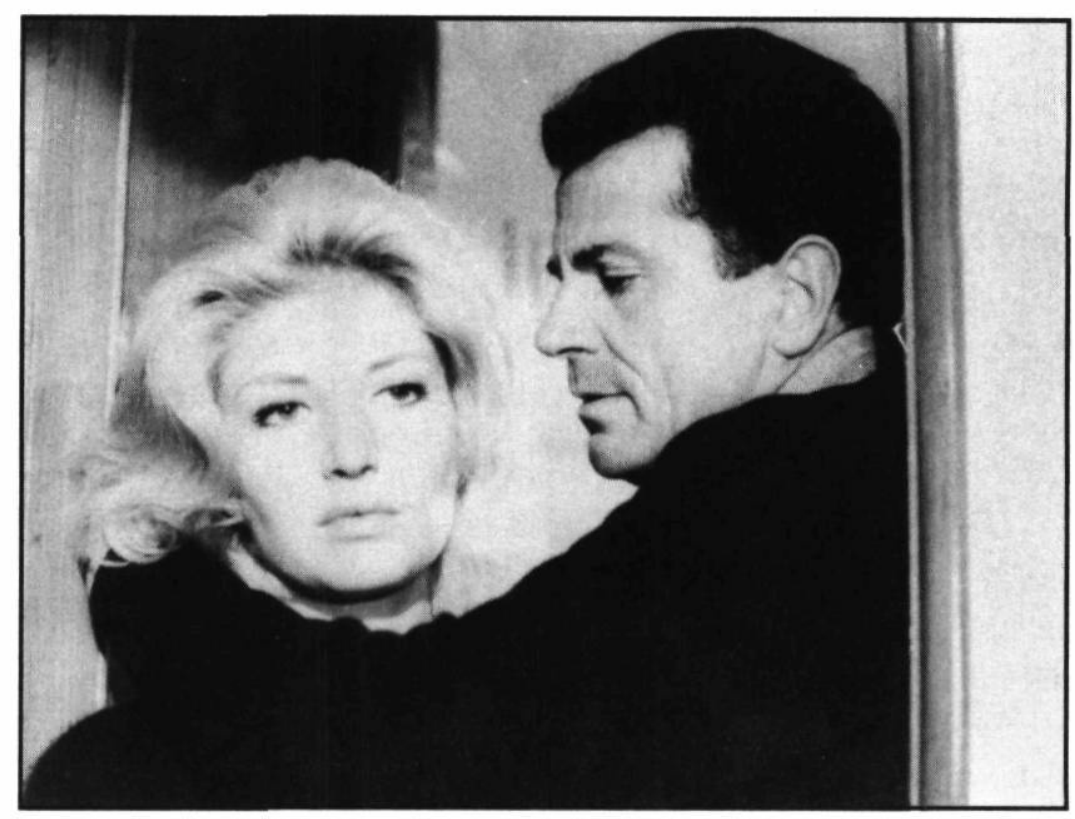

\section{L'Avventura de Michelangelo Antonioni (1960)}

son désir d'innover, de moderniser un langage filmique quelque peu ankylosé par des décennies de récits traditionnels.

Le travail de la presse française fait suite aux chroniques italiennes en considérant que L'Avventura est sans conteste le film le plus important de l'après-guerre, tout en le situant dans une continuité, celle établie par la littérature et la peinture qualifiées de "modernes». Parallèlement, les critiques suivent le "parcours de salle" du film et s'étonnent de la rapidité avec laquelle il acquiert son public, partout en France.

Enfin, on verra comment la critique anglo-saxonne au demeurant indifférente, sceptique, fort pragmatique, donnera lieu à des chroniques assez pertinentes ${ }^{3}$.

Guido Aristarco, qui préside depuis toujours aux destinées de la revue Cinema Nuovo, est celui qui s'attachera aux sources littéraires et culturelles du nouveau chroniqueur des "formes structurelles de l'âme». En l'ayant déjà fait pour Visconti, la piste est tracée, il ne lui reste plus qu’à fixer les jalons. Et ce n'est pas uniquement de littérature moderne, d'avant-garde, qu'il sera question dans l'essai d'Aristarco. Pour ce dernier, le concept de 
vie et d'art tel qu'il est exprimé avec justesse par Flaubert dans L'Éducation sentimentale est un point de départ pour Antonioni, avant qu'il ne débouche sur les expériences de l'avant-garde littéraire :

\begin{abstract}
Dans le sillage de Flaubert, il [Antonioni] semble ne pas comprendre ceux qui suivent la tradition du réalisme critique, il rejette toute extrême dialectique en donnant forme à des "personnages", il renonce à toute thèse, à toute intervention indirecte dans les événements et à toute interprétation directe des faits: il ne participe pas, il ne raconte pas, mais il observe et décrit la vie quotidienne monotone, régulière, plate. Son "roman" n'est pas, comme celui de Visconti, une progression dramatique et un récit au mécanisme parfait: il remplace cette forme par un récit où le protagoniste est le Temps $[\ldots]^{4}$.
\end{abstract}

Au cours de ce même essai, afin de valider le bien-fondé de ses rapprochements littéraires, Aristarco reprend les propos du cinéaste qui, lors de la publication du scénario, définit les nouvelles formes d'une éducation sentimentale:

Naturellement, les ambitions de L'Avventura sont plus vastes que celles d'un simple roman policier. Par exemple, cela peut vouloir dire que les sentiments auxquels la rhétorique sentimentale et l'histoire événementielle accordent aujourd'hui un poids définitif sont en réalité fragiles, insidieux, réversibles... Les camarades d'Anna commencent la recherche, surtout l'un d'eux, Sandro, l'amoureux. Cependant, à la moitié du film, le spectateur doit désirer que la jeune fille ne soit jamais retrouvée. Anna a laissé un vide, le vide lentement se combles

Lorsqu'il est enfin question de filiation avec les écrivains du $\mathrm{XX}^{e}$ siècle, c'est de Proust, de Joyce et de Camus qu'Antonioni semble être le plus proche, car il ne lui importe pas de caractériser la personnalité d'un protagoniste, mais plutôt d'analyser le mécanisme psychique, en soi. Ce type de remarques rejoint celles que le cinéaste formule à la même époque dans un débat désormais célèbre, publié dans la revue Bianco e Nero et portant le titre significatif "La maladie des sentiments»: 
J'ai décidé qu'il était important de montrer non pas les rapports entre personnages, mais ce qu'il y a à l'intérieur d'un personnage, afin d'essayer de comprendre ce qui s'était passé après la guerre; tous ces éléments qui ont marqué les êtres mais dont l'évolution psychologique n'a pas encore été analysée.

L'autre motivation tient au style, à la lassitude ressentie depuis un certain temps devant des techniques de narration normales et conventionnelles. Il m'a semblé important de relever les instants secondaires, les trajets périphériques, les moments durant lesquels les personnages s'abandonnent à eux-mêmes.

La vérité de notre existence n'est pas mécanique, conventionnelle, artificielle, comme le montrent les histoires traditionnelles. La cadence de la vie n'est pas équilibrée, elle est soit lente, soit rapide et il y a aussi des moments de stase ${ }^{6}$.

Les tentatives de théorisation de ce nouveau langage vont plus loin, et démontrent, comme le fait le critique Fernando Tempesti dans un article publié en décembre 1960 et intitulé "Le langage de L'Avventura", la nécessité imposée au public de distinguer entre un langage "rationnel " et un autre, "irrationnel»:

Le niveau de l'histoire immédiate, non émotive, est celui rationnel, alors que dans la plupart de nos films c'est l'irrationnel qui finit par l'emporter... La plupart des critiques lui ont d'ailleurs reproché son manque d'émotivité, de vraie poésie. En littérature, il y a des précédents (Stendhal, Diderot), mais au cinéma c'est un pionnier?

Il n'en demeure pas moins que cette mise en évidence de la nouveauté cinématographique en accord avec la modernité littéraire n'est pas perçue de la même manière par tous les critiques, encore moins par les cinéastes, et de façon très tardive par le public. Toutefois, le fait n'est pas nouveau, comme le démontrent des articles de Bianco e Nero datant de 1957, lors de la sortie de Il Grido, donc bien avant le déclenchement du "phénomène $L A v$ ventura". Dans "Michelangelo Antonioni, symbole d'une génération ", Giambattista Cavallaro souligne que la critique du moment est toujours très désorientée face au cinéma d'Antonioni : 
[...] elle ne sait jamais s'il utilise un schéma romantique pour donner une certaine coloration à un discours néoréaliste, commettant ainsi de graves erreurs par rapport au sujet, ou si par contre ses films sont des variations sur des états d'âme et des rapports sentimentaux difficiles, dont les milieux, les atmosphères, les dialogues et les événements ne sont que l'organisation narrative, comme l'orchestration d'un motif déjà défini à l'avance ${ }^{8}$.

Les critiques de langue française s'avèrent tout aussi soucieux de révéler la nouveauté voire la modernité de L'Avventura, mais l'incident cannois rend le parcours explicatif plus sinueux, moins linéaire que celui tracé par les confrères italiens. Des signatures prestigieuses telles celles de Sadoul, Kast ou Doniol-Valcroze préferent les quotidiens et les hebdomadaires généralistes tels France Observateur ou L'Express aux mensuels spécialisés comme Les Cabiers du cinéma ou Positif. L'on remarquera d'ailleurs que ces derniers font parfois preuve d'un opportunisme critique, voire d'un manque de rigueur tout à fait inattendus.

L'enjeu explicatif, lorsqu'il est question d'Antonioni, est de taille, et la tentation de transformer un compte rendu de visionnement en véritable essai est grande et souvent difficile à mâ̂triser. Ainsi, Georges Sadoul propose " [...] d'arrêter tout net les propos sous peine d'écrire un véritable livre sur l'art d'Antonioni» et il se trouve qu'il n'a pas tort, car ses considérations pêchent par un trop grand désir de globalisation: "Si nouveau que soit son art, il l'inscrit dans le néo-réalisme entendu non comme une formule étroite mais comme un dénominateur commun à des genres, styles, tempéraments et auteurs bien divers et différents ${ }^{9}$."

Les chroniques de Doniol-Valcroze ou de Claude Choublier dans France Observateur du 15 septembre 1960 témoignent d'un radicalisme hors pair. Selon Valcroze: «[...] parler de longueurs au sujet de L'Avventura, c'est trouver interminable la phrase de Proust." De son côté, Choublier met l'accent sur la nouveauté stylistique du film:

Il ne s'agit pas de la belle photo au sens de Figueroas, c'est à dire d'un palmier irisé par le soleil couchant [...] il s'agit d'un emploi totalement nouveau du scope, 
d'une composition de l'image jouant beaucoup sur la profondeur de champ et qui par le cadrage établit les rapports entre les personnages. De la même manière qu'en un seul cadrage on reconnaît aussitôt la patte de Welles ou d'Eisenstein, ici, dès le premier plan, on est entraîné dans un monde qui ne peut être que celui d'Antonioni.

Ces propos rejoignent ceux défendus par Fabio Carpi dans un des premiers livres jamais consacrés à Antonioni, étude publiée, une fois de plus, avant la sortie de L'Avventura, en 1958: "Je pense qu'Antonioni ne pourrait pas faire un film qui ne soit un film bien à lui, même en le voulant ${ }^{10}$."

Malgré le handicap d'un retard de réception, la Belgique, où L'Avventura sort "à l'heure où La Notte reçoit à Paris un accueil triomphant ", réagit d'une manière semblable, tout en évoquant les problèmes de censure et de non-distribution. En témoignent les très bonnes chroniques de La Flandre libérale d'octobre 1961, qui portent la signature d'Henri Rosenblatt:

Un homme et une femme entreprennent un voyage dont l'objet s'estompe rapidement. Lente modification: l'amitié se mue en amour. Sujet abordé par 1000 films peut-être, mais qui nous donnaient le spectacle de cette modification alors qu'ici elle devient le film lui-même. Antonioni refuse toute dramatisation [...]. Avec ce film, le cinéma arrive peut-être aux portes de l'incommunicable. En niant le spectacle, en remettant en cause le septième art, Antonioni, comme les plus grands créateurs de notre époque, refuse d'accepter toutes les fausses évidences.

Toutefois, cela va sans dire qu'aussi bien en France qu'en Belgique, Antonioni est loin de n'avoir que des adeptes parmi les critiques désireux de maintenir leur complicité avec un large public, qui ne souhaitent que se divertir et pour lequel "modernité " rime souvent avec "non-sens" ou "ennui". À titre d'exemples, voici les commentaires de deux vétérans de la critique belge, Maguy et Pierre Thonon, dans L'Écho de la bourse de juin 1960 :

Nommé par antithèse L'Avventura alors qu'en deux heures et demie de projection, il ne s'y passe virtuelle- 
ment rien d'apparent [...] la méditation d'Antonioni n'est pas neuve ni révolutionnaire. C'est une vieille rengaine de la sagesse populaire que l'amour, tel qu'on le conçoit sous l'influence de la littérature [...], ne peut subsister longtemps dans cette forme tronquée, réduite au simple et assez pitoyable niveau d'appétit, d'échange sexuel.

Les commentaires d'un Jean Domarchi dans les Cabiers $d u$ cinéma de la même époque (juin 1960) semblent plus nuancés, quoique très proches de l'esprit des Thonon:

Antonioni développe une conception de la durée comparable à celle du roman moderne à partir de Proust. Toutefois, la tentative de transcrire cette durée dans les limites d'un film d'une longueur normale échoue, dans la mesure où la mise en scène n'est pas à la hauteur de cette prétention excessive. On aboutit à des temps morts qui rompent le rythme du film, sans nous donner pour autant la sensation d'une durée réelle, comparable à celle que procure l'existence vécue. De telle sorte que le film laisse une impression d'irritation et parfois de malaise (p. 39-41).

Dans un numéro de Positif qui remonte à mars 1961, Henri Crespi se montre plus audacieux, en reposant le problème de la réception d'un certain type de cinéma, non pas d'un film en particulier. Avant lui, lors de la présentation huée de L'Avventura à Cannes, d'autres collaborateurs du même mensuel, tels Nelly Kaplan ou Robert Benayoun, avaient avancé le même type d'affirmations mais avec moins d'audace. Ils mettaient notamment l'accent sur "des siècles d'éducation dirigée" et sur "l'ankylose de certaines infrastructures" :

J'ai lu quelques articles sur L'Avventura [...] l'on nous affirme que ceux qui ne s'aperçoivent pas que c'est un chef-d'œuvre sont des imbéciles patentés. C'est trop vite dit à mon goût. Je connais des gens fort intelligents qui n'ont pas aimé [...] j'en connais d'autres qui l'ont trop aimé.[...] L'œuvre d'art est faite pour l'amateur et non l'amateur par l'œuvre. L'Avventura est une œuvre d'avant-garde, je pense qu'il faudra quelque temps avant de pouvoir la recevoir à un degré d'émotion 
"supportable", avant que l'on puisse prendre un vrai plaisir à être dérangé par elle (p. 31-35).

L'accueil critique du monde anglo-saxon à L'Avventura revêt souvent une tonalité pragmatique non loin de celles citées plus haut. Lors de la présentation du film dans Variety, en mai 1960, le critique en question note dans le plus pur style télégraphique made in U.S. A. :

Pas de grosses chances de survie en dehors de circuits élitistes, car sur-emphatique et ayant besoin de beaucoup de coupures! [...] La difficulté vient aussi des personnages fort ambigus quoique bien interprétés. Seule voie possible: les séances spéciales.

Le mensuel américain Films in review (vol. 12, $\mathrm{n}^{\circ} 5$, mai 1961) offre l'exemple d'un compte rendu extrêment virulent, voire vulgaire et violent, qui mérite d'être cité intégralement. Il témoigne de la mentalité d'une certaine société d'outre-Atlantique qui s'est toujours insurgée contre tout ce qui n'est pas de l'entertainment au vrai sens du terme, en attaquant constamment "les intellectuels imposteurs" qui osaient produire des œuvres "où rien ne se passe" :

Le film est une imposture, le fait que Signor Antonioni prétend qu'il s'agit d'un "drame intérieur" et que le public doit "réfléchir " afin de comprendre, c'est de la vaste blague. Le fait est qu'Antonioni n'avait aucun scénario, qu'il n'a rien pu improviser de valable, et que toute cette pellicule insensée a réussi à s'imposer comme une soi-disant œuvre d'art aux yeux des producteurs.

A ce stade tout le monde a compris qu'il ne se passe absolument rien dans ce film [...]. Les personnages, si vraiment on peut les qualifier de personnages, ne font qu'errer dans tous les sens le regard vide de toute expression, pour la simple et bonne raison que le réalisateur ne leur a rien fourni [...]. Comment peut-on tolérer une telle imposture?

Eh bien, c'est parce que Antonioni a introduit deux longues scènes de baisers et a demandé, ne sachant que faire d'autre, à ses actrices d'enlever leurs blouses [...]. 
La Gauche européenne a soutenu le film en argumentant qu'il s'agissait d'une "critique de la décadence de la grande bourgeoisie italienne». Je pense que Marx, et plus encore Lénine, l'aurait plutôt qualifié de "chronique de la décadence du réalisateur Antonioni " (p. 295).

À mille lieues du parti pris réactionnaire affiché par Ellen Fitzpatrick dans l'article cité plus haut, les opinions de la rédactrice de Sight and Sound, Penelope Huston, montrent bien qu'en Angleterre, les choses se passent d'une manière quelque peu différente. C'est d'ailleurs à l'intérieur des pages de Sight and Sound que l'on trouvera, au fil des années, une remarquable série d'articles consacrés à l'œuvre antonionienne. En évoquant l'incident cannois, Huston insiste en 1960 sur l'attitude négative du public (bâillements, rires), sans précédent à son avis, dans l'histoire du festival. Néanmoins, ce phénomène ne semble pas l'étonner, car elle le généralise à l'échelle du monde entier. Malgré cet échec de communication avec le public, "[...] on échangerait bien - écrit-elle - L'Avventura contre six succès faciles". Pour Huston, qui considère ce film tout aussi pionnier que le Hiroshima de Resnais :

Antonioni fait de la philosophie à l'écran : il se concentre sur la vacuité de l'existence, sur l'incapacité d'action et sur la léthargie et les frustrations d'une certaine société. Le film installe une monotonie délibérée, fait impardonnable aux yeux du public. Le récit est démembré, il y a un décalage incompréhensible entre la première partie, assez homogène (la disparition et le début de la recherche) et la deuxième, entropique (le désordre d'un amour incertain) (p. 121-122).

En procédant ainsi au dépouillement systématique des critiques italo-françaises et anglo-saxonnes de l'époque, l'on arrive à mieux cerner la problématique de la modernité antonionienne. Celle-ci semble correspondre à une rupture, à une faille à l'intérieur des structures esthétiques existantes, mais surtout, et ceci a sans doute quelque chose de paradoxal, au niveau des structures sociologiques. Les chroniqueurs de L'Avventura évoquent ses aspects novateurs, sa parenté avec la littérature (et plus 
tard, avec la peinture) moderne, sa difficulté inhérente de compréhension. Néanmoins, ils aboutissent presque toujours à des considérations d'ordre sociétal, qui passent par une remise en question de la réception spectatorielle. C'est ce que définira plus tard Freddy Buache, dans son étude consacrée au cinéma italien, comme étant le "refus de conditionnement des réactions du spectateur, au sens pavlovien du terme".

Pierre Kast fait aussitôt remarquer, dans L'Express, le fait que "[...] quelque chose est en train de se passer dont le succès de salle de la rétrospective Antonioni est une première manifestation" (nous sommes le 29 septembre 1960 !). Parallèlement, dans les pages des Cahiers du cinéma cette fois-ci, DoniolValcroze parle du "facteur rhésus et du nouveau cinéma". Il range L'Avventura aux côtés d'Hiroshima mon amour, en soulignant que ces deux films "[...] inaugurent ce qu'il va bien falloir nommer par son nom: "le nouveau cinéma" " (p. 47-49).

Ce genre de considérations ne sauraient épuiser, à l'intérieur d'un quotidien ou d'un hebdomadaire une telle problématique: une réponse relativement exhaustive au défi socio-esthétique lancé par le nouveau type de cinéma véhiculé par L'Avventura se trouve dans l'essai désormais célèbre que Marie-Claire Ropars publie dans la revue Esprit, en décembre 1960. Dans son remarquable essai, il est question de nouvelles images, mais surtout de nouveaux rapports interindividuels, rapports que le spectateur devrait pouvoir suivre en tenant compte de leur statut évolutif, non finalisable:

\begin{abstract}
L'absence de bonheur de l'individu vient du décalage entre les mythes préexistants et le monde moderne. Limpossibilité des couples de communiquer a ses origines dans un malaise plus profond, qu'ils reflètent en même temps que ceux qui les entourent. Chaque scène est donc construite en profondeur, afin de montrer aussi bien les protagonistes que leur image reflétée [...] L'histoire pose une question sans pour autant la résoudre; le labyrinthe n'a pas d'issue, c'est l'itinéraire qu'il faut examiner.
\end{abstract}

Si l'on s'en tient à la comparaison initiale avec la critique italienne, qui possède, par la force des choses, plus d'éléments "à 
l'appui ", il est intéressant de noter que l'impact de L'Avventura n'est pas considéré comme un cas isolé; Aristarco, Carpi et bien d'autres évoquent constamment dans leurs analyses des passages significatifs de Cronaca di un amore, de Il Grido, voire de certains courts métrages. Ils démontrent avec objectivité que ce qui est considéré comme étant nouveau ne l'est pas entièrement. D'autres, parmi lesquels le jeune Pasolini, sont même très critiques à l'égard de cette nouvelle vision des rapports humains et du monde filmique qui les entoure.

À l'occasion d'un questionnaire envoyé à la fin de l'année 1960 par la revue Cinema Nuovo à plusieurs écrivains et critiques, les avis semblent très partagés. Voici quelques-unes des réponses à la question: "Quel est le réalisateur italien le plus important du moment?»:

Luigi Russo: J'ai le sentiment que les personnages d'Antonioni doivent avoir plus de "chair" et qu'il faut remplir les plages de silence; sinon on se limite à une esthétique en point d'exclamation.

Galvano della Volpe: Je préfere L'Avventura à La Dolce Vita, à cause de sa nouvelle poétique, mais j'attends toujours un film libre de l'esprit décadent.

Pier Paolo Pasolini: L'existentialisme d'Antonioni est inculte et donc un peu provincial. Seule une analyse marxiste de l'angoisse est actuelle. Il ne suffit pas, comme le fait Antonioni, de témoigner [...] Ceci mis à part, je le considère comme un des auteurs les plus raffinés et sincères de ces dernières années. Pour moi, c'est Fellini le plus génial de nos cinéastes, car son monde stylistique est inconfondable ${ }^{11}$.

La réponse de Pasolini n’a rien de surprenant vu le courant marxisant fort en vogue dans la critique italienne de l'époque, une des figures de proue de ce courant étant Aristarco luimême. Aux yeux d'Aristarco, la triade constituée par L'Avventural La Nottel L'Eclisse serait " [...] intéressante du point de vue artistique ", mais décadente et de ce fait irrationnelle :

Antonioni est certes un moraliste qui lutte contre la morale courante, conventionnelle, contre les préjugés bourgeois, mais au nom d'une liberté à laquelle lui- 
même ne croit sans doute plus. Comme Ibsen, il est un révolutionnaire sans idéaux sociaux; et le réformateur risque de se transformer, ou plutôt s'est déjà transformé, en un fataliste désespéré ${ }^{12}$.

Étudier le film d'un point de vue moral, en essayant de décrire les mœurs individuelles plus que l'architecture visuelle, c'est donc ce qui intéresse le plus les critiques chez Antonioni "le moderne", ne fût-ce que pour l'avantage du débat d'idées qui peut en découler.

Toutefois, il ressort à travers la comparaison de plusieurs courants d'opinion que le fait de privilégier l'aspect moral ne saurait épuiser le film; l'on constate d'ailleurs que le côté purement esthétique échappe souvent à l'attention critique. Cette omission ne s'avère pas, tout compte fait, complètement innocente: elle a ses motivations dans la volonté des critiques du moment de s'approprier la théorisation d'un nouveau type de cinéma, alors que le film demeure le pur produit de son auteur et de ses coscénaristes (il ne faudrait pas passer sous silence le rôle déterminant de Tonino Guerra et d'Elio Bartolini dans le façonnage de ce récit abstrait). Un appoint de modestie et d'humilité aurait sans doute contribué à la création d'une plus grande synergie entre l'opinion critique et celui qui a "fabriqué" une œuvre jugée si avant-gardiste.

Comment expliquer le fait qu'il est si peu question de la perception d'une nouvel univers visuel, bien plus proche du cinéma documentaire et de certains aspects de la peinture moderne que de la littérature évoquée à tout instant? Certes, Antonioni luimême en tant qu'ancien critique littéraire et cinématographique s'est méfié, dès le début, des interprétations esthétiques hasardeuses. Lors de la conférence de presse autour de L'Avventura, à Cannes, il a plutôt mis l'accent sur " [...] la grande fracture entre une science entièrement projetée vers le futur et une moralité rigide et sévère $[\ldots]$ ".

Pourtant, à y regarder de plus près, les éléments critiques englobés dans la démarche de l'auteur sont lisibles dès 1959 dans sa confession-manifeste "Fare un film è per me vivere». Le cinéaste évoque, entre autres, sa première expérience de tournage à Ferrare, avant la guerre. Il s'agissait de filmer sur le vif, coûte 
que coûte, les malades d'un asile. Cette expérience l'avait marqué non pas par sa terrible véracité, mais par les effets qui en découlaient au niveau de sa propre expérience visuelle et mentale. C'est ainsi qu'il écrit, semblant contredire les propos d'Aristarco:

Faire un film, cela ne ressemble pas à la rédaction d'un roman. Flaubert disait que vivre n'était pas son métier: son métier était d'écrire. Faire un film, c'est au contraire vivre, du moins pour moi. Mon histoire personnelle ne s'arrête pas pendant les prises de vue d'un film: au contraire, c'est alors qu'elle devient plus intense [...]. Le cinéma qui me plaît est celui dans lequel les images apportent un sens de vérité sans perdre leur force de persuasion, sans infatuation ni délire ou extravagance intellectuelle: les choses vues en face, ni à l'envers, ni par-derrière, ni en biais [...] J'ai très peur du public et même des critiques. Je voudrais pouvoir les prévenir, leur expliquer une quantité de choses avant qu'ils voient un de mes films ${ }^{13}$.

En remontant dans le temps pour retrouver grâce à une publication ultérieure les Écrits de jeunesse du réalisateur, les germes d'une pensée visuelle complexe sont facilement décelables. En 1942, Antonioni signe dans la revue Cinema, un article sur les "Suggestions de Hegel». En voici l'un des passages les plus représentatifs :

Le cinéma est un art fondamentalement figuratif, et comme la peinture, son moyen de représentation formelle est l'apparence extérieure de la nature et des individus, à condition que celle-ci laisse clairement deviner leur intériorité. Remarquez que je dis bien apparence et non pas matière; un rapport précis entre la spiritualité et la sensibilité est donc indispensable [...].

Durant les années qui suivent, au gré de ses collaborations au Corriere Padano ou à L'Italia libera, le réalisateur livre aux lecteurs aussi bien des descriptions précises et poétiques d'endroits et d'habitants de Ferrare que des critiques de films qui témoignent d'un style franc et d'une absence totale de complaisance.

Comme le soulignent à plusieurs reprises dans leurs exégèses «bilans» Gian Piero Brunetta et Giorgio Tinazzi, Antonioni 
participe à l'aventure critique et poétique de l'après-guerre, mais il le fait en se concentrant sur la productivité des significations visuelles, non pas sur la force autosignifiante des contenus. Les recensions de Visconti, Carné (dont il fut aussi l'assistant), Olivier ou Walsh démontrent à quel point la mise en question des modalités traditionnelles du récit visuel était dès le début au centre de ses préoccupations. Les références constantes à la peinture laissent entrevoir les pistes visuelles que le futur cinéaste empruntera plus tard. Braque et Matisse semblent être, dans la peinture française, des points de repère; dans sa recension d'Ivan le terrible, il compare "les hurlements visuels" à la peinture expressionniste; enfin, lors de la sortie de Henry $V$ d'Olivier, il remarque un lien entre les images du film et les peintres flamands.

En se référant à L'Avventura, les auteurs de véritables études critiques évoqueront à leur tour d'autres influences picturales qu'Antonioni ne cite jamais explicitement, mais qui l'ont incontestablement marqué. Il s'agit de Morandi, de De Chirico et de l'hyperréaliste Edward Hopper, tous préoccupés par une nouvelle dynamique de l'individu à l'intérieur d'un espace arbitraire et illimité. De nos jours, le cinéaste lui-même pratique la peinture, comme en témoignent les albums et les différentes expositions qui lui ont été consacrés.

Arrivés à ce point de notre survol critique des deux "camps", celui des journalistes-écrivains et celui de l'auteur, on pourrait donc se demander pourquoi ce repli momentané des chroniqueurs sur l'avant-garde sociolittéraire et non pas sur le véritable terrain du visuel, plastique ou purement cinématographique? S'agit-il d'un manque de préparation, à porter sur le compte de la difficulté de lecture d'une œuvre abstraite, de la nécessité déjà invoquée de laisser passer le temps, ou bien comme le craignait Antonioni, d'une mauvaise compréhension des véritables intentions de l'auteur?

Deux réponses s'imposent: l'une liée à l'actualité du moment, à savoir la publication du scénario commenté de L'Avventura, en cette même année 1960; l'autre livrée par un essai d'Alain Bergala paru dans les pages de la revue Cinémathèque en 1992 et faisant écho aussi bien aux chroniques de Serge Daney qu'aux 
théories développées par Gilles Deleuze et par Seymour Chatman au sujet de la modernité antonionienne.

Lors de la publication du scénario et des dialogues de L'Avventura (dont la traduction française, chez Buchet-Chastel, est quasi simultanée), le critique italien Tommaso Chiaretti se fait l'interprète des pensées du cinéaste. Dans "Antonioni ou le refus de la banalité ", Chiaretti met en garde dès le début ceux qui seraient tentés de pratiquer l'amalgame du cinéma avec le roman, l'art figuratif et la musique, car " [...] ce n'est qu'un masque destiné à cacher parfois le manque d'imagination", et ceci ne peut être le cas pour Antonioni. Il reconnaît que des intérêts communs peuvent exister entre un auteur de littérature et un auteur de cinéma, mais il s'efforce de démontrer à quel point l'histoire de la lutte menée pour la réalisation de L'Avventura témoigne de son originalité et de sa spécificité cinématographiques. Les conditions difficiles de tournage et d'achèvement du film, les détails sur l'abandon du producteur et de la première équipe, débouchent sur un résultat paradoxal, car les prouesses techniques au niveau du son et de l'image semblent avoir été réalisées dans un contexte "sans nuages". Selon Chiaretti :

Le problème fondamental de L'Avventura n'est qu'en apparence un problème technique, de découpage. Il s'agissait de renverser la situation sans faire violence au spectateur. De lui faire vivre totalement l'expérience des deux héros, de faire en sorte que lorsque Sandro et Claudia "oublient" l'existence même d'Anna, le spectateur ait lui aussi oublié, ait perdu tout intérêt pour une enquête policière [...] L'Avventura donne profondément tort à ceux qui attribuent à Antonioni un style "à tout faire", profondément codifié. Ici, les longs cadrages ont souvent été remplacés par le montage rapide de courts passages. Il se dégage en réalité un ton tellement peu d'avant-garde, dans le pire sens du terme, qu'il frappe immédiatement. Le montage de certains cadrages de type documentaire sur l'île rappelle sans aucun doute Flaherty. Quel est donc le style d'Antonioni? Chez Antonioni, le style, c'est la rigueur à travers la recherche du langage le plus exact, «irremplaçable", pour exprimer un monde ${ }^{14}$. 
L'exégète privilégie par conséquent la piste d'une nouvelle forme de réalisme visuel qui relève parfois du documentaire par son hyperréalité, mais qui demeure une œuvre filmique entièrement personnelle, dont les intentions de signification sont posées rigoureusement, sans pour autant qu'elles soient transparentes pour tout le monde. C'est pour cela que Chiaretti ne semble pas d'accord avec toutes sortes d'étiquettes "collées" au cinéaste par facilité: celle de néo-réaliste tendance bourgeoise, de critique marxiste de la crise des rapports entre les sexes ou encore d'héritier de Pavese, qu'Antonioni connaît bien mais dont il refuse la filiation, invoquant plutôt avec lui une association culturelle.

D'un point de vue pédagogique et non pas strictement informatif, il était néanmoins difficile pour la critique de l'époque de saisir l'unicité d'une démarche qui se veut rigoureuse et claire, alors que, au niveau de la narration du moins, elle transgresse les critères classiques de vraisemblance de l'action, ainsi que certains paramètres spatio-temporels (étirement du temps, personnages désorientés dans l'espace, etc.).

La critique récente du cinéma d'Antonioni nous offre une solution interprétative qui illustre à quel point la modernité mise en place par ses films relève des conceptions de l'auteur, tout en nécessitant une contextualisation critique, que seul le recul des années a pu engendrer. Il est vrai que Bergala se concentre, dans son article, sur l'Antonioni "des années 50 ", refusant de se limiter à un seul film. Ceci ne fait que confirmer les thèses dont il était question au début de cet article, et notamment celle de Fabio Carpi. Cette concentration sert à inscrire Antonioni dans « la deuxième vague de modernité, l'après aprèsguerre". Alors qu'il n'y a que six ans de différence entre le cinéaste et Rossellini, ils font partie de générations distinctes:

Antonioni repart d'un état du cinéma antérieur au néoréalisme en se référant à une conception somme toute classique du scénario, pour y tracer ses propres ruptures, ses propres marques [...]. Le point de départ de L'Avventura, œuvre-manifeste, c'est justement l'effacement: une femme disparaît, sans laisser de traces (apparentes!); des questions essentielles (où est le per- 
sonnage?; comment est-il arrivé là; combien de temps s'est écoulé depuis... ?) finiront par trouver de façon plus ou moins différée et fractionnée leurs réponses, mais au terme d'un temps de latence où le spectateur doit faire face à l'énigme du nouveau plan et de la chose simplement montrée ${ }^{15}$.

On arrive ainsi à ce que l'exégète qualifie "d'esthétique de l'oubli "; à un discours qui montre pour mieux cacher, rappelant en quelque sorte l'auto-évidence trompeuse d'un Magritte, mais laissant aussi la porte ouverte vers l'infini, à la manière d'un Brancusi.

La difficulté de saisie critique du phénomène L'Avventura vient de son unicité, de son abstraction, mais aussi du non-dit qu'Antonioni désirait installer : il suffit de se remémorer l'l̂le mystérieuse, les silences de Claudia / Vitti, le regard perdu de Sandro / Ferzetti à la fin du film, à l'heure d'un hypothétique pardon.

Lorsqu'en 1983, L'Avventura ressort en France sur les écrans de télévision (bien après des œuvres antonioniennes plus fréquemment diffusées comme Blow up ou Professione Reporter), Jacques Siclier affirme avec beaucoup d'assurance: "Son style psychologique, ses temps morts, ne peuvent plus déconcerter." Nous ne pouvons qu'espérer qu'il ait raison; toutefois, il est à craindre que le récit de L'Avventura, voire son style risquent encore de déconcerter un public pour lequel la modernité cinématographique demeure un phénomène unique, irrépétable, isolé.

Bien sûr, il y a Wim Wenders, et son cinéma poursuit à bien des égards les leçons du maître, avec lequel il a d'ailleurs entamé un projet cinématographique commun. Mais à la différence des anges dans Les Ailes du désir, les démons de la postmodernité encombrent le ciel cinématographique européen et empêchent cinéastes et critiques de défricher un chemin encore vierge, de repenser l'image, comme l'avait fait, au risque d'être hué et incompris, Michelangelo Antonioni.

Université libre de Bruxelles 


\section{NOTES}

1 Cet article doit beaucoup à la série de cinq volumes consacrés à Michelangelo Antonioni et reprenant la critique de ses films, ses entretiens, ses écrits, ses projets non réalisés ainsi qu'une biblio-filmographie complète. Cette entreprise de publication s'est faite sous l'égide de Cinecittà International et du ministère italien du Tourisme et du Spectacle (entre 1991 et 1995) sous la direction de Carlo di Carlo. Qu'il en soit ici, une nouvelle fois, vivement remercié.

2 Michelangelo Antonioni: Identificazione di un autore (collectif), vol. 1. Parme: Pratiche, 1983.

3 Il est bon de rappeler aux lecteurs que les ouvrages en langue anglaise consacrés ultérieurement à l'œuvre de Michelangelo Antonioni, à savoir ceux de P. Strick, P. Cowie, S. Chatman ou E. Rifkin, s'avèrent des outils critiques et pédagogiques indispensables.

4 Aristarco, Guido. "L'univers sans qualité". Cinema Nuovo, n 157 (1962) p. 190-198.

\section{Idem.}

6 Extrait du colloque organisé le 16 mars 1961 au Centro Sperimentale di Cinematografia de Rome et publié dans Bianco e Nero, vol. 22, n 2-3 (février-mars 1961) p. 69-95.

7 Tempesti, Fernando. "Le langage de L'Avventura". Cinema Nuovo, $\mathrm{n}^{\circ} 148$ (novembre-décembre 1960) p. 490-491.

8 Cavallaro, Giambattista. "Michelangelo Antonioni, symbole d'une génération ". Bianco e Nero, vol. 18, $\mathrm{n}^{\circ} 9$ (septembre 1957) p. 17-56.

9 Coupure de presse non identifée, figurant dans le dossier de presse de L'Avventura rassemblé par la Cinémathèque royale de Belgique.

10 Carpi, Fabio. Antonioni. Parme: Guanda, 1958, p. 16-17.

11 Voir à ce sujet le numéro 151 de la revue Cinema Nuovo, paru début 1961.

12 Voir la revue Cinema Nuovo de mai-juin 1962.

13 Voir à ce sujet la traduction française de l'article parue dans la revue Premier Plan, $\mathrm{n}^{\circ} 15$ (décembre 1960) p. 74-76.

14 Voir l'introduction au scénario/découpage de L'Avventura (Paris: Buchet/Chastel, 1960).

15 Carpi, Fabio, op. cit., p. 26. 